\title{
Ergebnisse zur forschungsbasierten Entwicklung, Implementation, Evaluation und Weiterentwicklung eines Fortbildungsangebotes für Lehrkräfte zum »Professionellen Umgang mit heterogenen Lerngruppen «
}

\section{Christina Baust \& Anita Pachner}

Journal für Psychologie, 27(2), 263-287

https://doi.org/10.30820/0942-2285-2019-2-263

www.journal-fuer-psychologie.de

\section{Zusammenfassung}

Im Rahmen des Beitrags wird der Frage nachgegangen, inwiefern eine Lehrerfortbildung zum »Professionellen Umgang mit heterogenen Lerngruppen « heterogenitätssensibles Handeln von Lehrkräften zu fördern vermag. Dazu wurde die Fortbildungspraxis mithilfe von Beobachtungsprotokollen und verschriftlichten Reflexionsaufgaben aus zwei Fortbildungsrunden systematisch erfasst. Mithilfe einer inhaltsanalytischen Auswertung (Mayring 2015) und anschließender Triangulation dieser beiden Datenquellen konnten die Lernprozesse der Teilnehmenden nachgezeichnet werden. Dabei bildete die forschungsbasierte Entwicklung, Implementation, Evaluation und Weiterentwicklung des Fortbildungsangebotes nach dem Design-Based Research Ansatz den Rahmen der Studie. Ein zentrales Ergebnis sind die unterschiedlichen Ausprägungen der Bewusstheit der eigenen Beobachtungs- und Handlungspraxis der Lehrkräfte sowie die Ausprägungen der Bereitschaft, die Beobachtungs-und Handlungspraxis weiterzuentwickeln. Die Teilnehmenden differenzieren diese Ausprägungen im Verlauf der einzelnen Fortbildungsphasen aus.

Schlüsselwörter: Professionalisierung von Lehrenden, Inklusion und Heterogenität, DesignBased Research, Reflexion, Perspektivverschränkung, Lehrerfortbildung, Lernprozesse in der Erwachsenenbildung 


\section{Summary}

Findings of the research-based development, implementation, evaluation and further development of a teacher training series on »The Professional Handling of Heterogeneous Learning Groups"

In this article, the following question is examined: To what extent fosters a reflection-centered teacher training on »The Professional Handling of Heterogeneous Learning Groups « the sensitive handling of heterogeneous learning groups. To answer this question, the interactions within the teacher training were systematically recorded by means of observations protocols and by written reflection tasks. On the basis of content analyses (Mayring 2015) and the subsequent triangulation of these two data sources, the learning processes of the participants could be traced. There will also be a focus on the research-based development, implementation, evaluation and further development of the teacher training, derived from the DesignBased Research approach. One of the main findings are the different characteristics of the teacher's own observation-and action-practice in class and the different characteristics of the willingness to develop this observation- and action-practice. The participants develop these characteristics in the course of the different phases of the training offer.

Key words: professional development of teaching professionals, inclusion and heterogeneity, Design-Based Research, reflection, interaction, teacher training, learning processes in further education

\section{$1 \quad$ Einleitung}

Der berufsbegleitenden Professionalisierung von Lehrkräften kommt aufgrund der hohen inneren Dynamik, Unsicherheit und wachsenden Vielfalt des Berufsfeldes eine immer größere Bedeutung zu (vgl. Schön 1983, Terhart 2015). So erleben viele Lehrkräfte den Umgang mit und das Ermöglichen von Heterogenität und Differenz im schulischen Zusammenhang als Verunsicherung und Herausforderung, was mit einem hohen Bedarf an Fortbildungsangeboten zu diesem Thema einhergeht (vgl. Biederbeck und Rothland 2017). Die Erwachsenenbildung ist hier gefordert, mit innovativen Lernarchitekturen und Lernbegleitungen zu antworten, die professionelles Lehrhandeln im Umgang mit heterogenen Lerngruppen fördern können (vgl. Siebert 2012, Terhart 2015). So gilt es als Voraussetzung für heterogenitätssensibles Lehrhandeln, dass die Lehrkräfte für die Bedeutung ihrer Beobachtungs- und Handlungspraxis sensibilisiert sind und sich reflexiv mit der eigenen Unterrichtspraxis auseinandersetzen, um diese weiterentwickeln zu können (vgl. Emmerich und Goldmann 2018). Dazu eigenen sich in besonderem Maße reflexionszentrierte Lernumgebungen, in denen die Teilnehmenden dabei unterstützt werden können, ihre Handlungspraxis theoriegeleitet 
und im Austausch mit anderen Professionellen zu analysieren und weiterzuentwickeln (vgl. Hof 2007; Helsper 2016). Für die empirisch fundierte Gestaltung solcher Fortbildungsangebote fehlen jedoch noch Erkenntnisse darüber, wie Lernprozesse im Rahmen solcher Lernumgebungen verlaufen und inwiefern heterogenitätssensibles Lehrhandeln tatsächlich angeregt werden kann.

Um dieser Frage nachzugehen wurde in einem Projekt der BMBF-geförderten »Qualitätsoffensive Lehrerbildung « ${ }^{1}$ ein Fortbildungskonzept zum »Professionellen Umgang mit heterogenen Lerngruppen « von einem interdisziplinären Team aus Erwachsenenbildung und Allgemeiner Pädagogik mit Schwerpunkt Inklusion, Heterogenität, Diversität konzipiert und realisiert. Im Zuge der Evaluationsstudie zu dieser Lehrerfortbildung konnten die Lernprozesse der Teilnehmenden im Rahmen zweier Fortbildungsrunden nachgezeichnet werden. Dabei waren die beiden Fragestellungen leitend, inwiefern ein Bewusstsein für die eigene Beobachtungs- und Handlungspraxis im Umgang mit Heterogenität geschaffen werden konnte und inwiefern das auch mit einer Veränderung dieser einhergeht. Um Erkenntnisse über die lernförderliche Gestaltung und Implementation von reflexionszentrierten Lernumgebungen zum Umgang mit Heterogenität zu gewinnen, war weiter die Frage elementar, ob das Fortbildungsangebot den Bedarfen der Lehrkräfte entspricht. Im Sinne des Design-Based Research Ansatzes soll die Studie dabei sowohl zur Entwicklung innovativer Lösungen für Herausforderungen der Bildungspraxis als auch zur Generierung wissenschaftlicher Erkenntnisse beitragen (vgl. Design-Based Research Collective 2003) ${ }^{2}$. Der Beitrag ist dementsprechend entlang der Forschungs- und Entwicklungsphasen des Design-Based Research Ansatzes aufgebaut (vgl. Euler 2014b): Auf Basis einer differenzierten Zielund Problempräzisierung aus praktischer und theoretischer Perspektive (Abschnitt 2.1) folgen die aus zwei Zyklen bestehende Entwicklung, Erprobung, Evaluation und Verfeinerung des Designs (Abschnitt 2.2 bis 2.5). Das Vorgehen zielt ab auf die Generierung von Gestaltungsprinzipien (Abschnitt 2.6), die abschließend kritisch diskutiert werden (Abschnitt 3).

\section{Design-Based Research - Die Forschungs- und Entwicklungsphasen des Fortbildungskonzeptes}

Die gestaltungsorientierte Forschung gemäß Design-Based Research zeichnet sich durch eine fortwährende Anwendungsorientierung, den kontinuierlichen Einbezug von Praktiker*innen, die theoriegeleitete Verankerung des Forschungs- und Gestaltungsprozesses sowie den integrativen Einsatz von Forschungsmethoden aus. Dabei werden Forschung und Entwicklung als iterative und zirkuläre Prozesse konzipiert (vgl. Raatz 2016). Nach dem Prozessmodell von Euler (2014b) beginnt dieser Forschungs- und Entwicklungs- 
prozess mit einer differenzierten Problem- und Zielpräzisierung, in die sowohl die praktische als auch die theoretische Perspektive auf den Gegenstand einbezogen werden: $\gg$ Dies erfolgt zum einen durch die Identifikation relevanter Theorien und die Formulierung theoriebasierter Annahmen für die Gestaltung der Intervention (theoretische Perspektive), zum anderen durch eine Kontextanalyse sowie die Aktivierung oftmals impliziten Erfahrungswissens aus der Praxis (praktische Perspektive) (Raatz 2016, 43).

\subsection{Die Professionalisierung von Lehrkräften zum Umgang mit heterogenen Lerngruppen - Ziel- und Problempräzisierung aus praktischer und theoretischer Perspektive}

Gemäß dem empirisch fundierten Angebots- und Nutzungsmodell zur Erklärung der Wirksamkeit von Lehrerfortbildungen nach Lipowsky (2010) hängt die Entwicklung der Teilnehmenden im Rahmen von Fortbildungs- und Professionalisierungsmaßnahmen von unterschiedlichen Faktoren ab: Dies sind die individuellen Voraussetzungen der Teilnehmenden, die kontextuellen Bedingungen sowie die strukturellen und didaktischen Merkmale der Fortbildungen. Dabei beeinflussen die Merkmale des Fortbildungsangebotes die Wahrnehmung und Nutzung des Angebotes durch die Lehrkräfte. Die Wahrnehmung und Nutzung des Angebotes durch die Teilnehmenden ist wiederum ausschlaggebend für den Transferprozess und den davon abhängigen Fortbildungserfolg (vgl. Lipowsky 2010). Im Rahmen der vorliegenden Evaluationsstudie soll dieses Zusammenspiel von Fortbildungskonzept und Wahrnehmung bzw. Nutzung des Angebots durch die Lehrkräfte in den Blick genommen werden. Dazu wird das Fortbildungsangebot sowohl auf Ebene der Zufriedenheit der Teilnehmenden als auch auf Ebene der Lernprozesse evaluiert (vgl. Lipowsky 2010).

\subsubsection{Praktische Perspektive}

Für die Entwicklung und Implementation von Bildungsinnovationen ist die Perspektive der Praxisakteure entscheidend: Zum einen können sie wertvolles, implizites Erfahrungswissen einbringen und dazu beitragen, dass die Intervention Anwendung in der Praxis finden kann. Zum anderen sind die Praxispartner*innen - in diesem Fall die Lehrkräfte - Adressat*innen der Bildungsinnovation, das heißt, die Teilnahme am Fortbildungsangebot hängt davon ab, ob dieses von ihnen als bedarfsgerecht wahrgenommen wird (vgl. Euler 2014b; Schmidt-Hertha und Werner 2019). Um das Fortbildungsangebot gemäß den Bedarfen der Lehrkräfte zu gestalten und dabei außerdem den Kontext der Innovation zu berücksichtigen, wurden zu Beginn des Projektes »Lehrerfort- und - 
weiterbildung « der BMBF-geförderten Tübingen School of Education folgende Schritte durchgeführt: Zuerst wurde das gesamte Lehrerfort- und -weiterbildungsangebot in Baden-Württemberg hinsichtlich bestehender Schwerpunkte und Lücken analysiert. Hier zeigte sich »eine deutliche Dominanz kurzer, meist halbtägiger Veranstaltungen, deren Nachhaltigkeit auf Basis des aktuellen Forschungsstands zumindest infrage gestellt werden muss « (Schmidt-Hertha und Werner 2019, 254; vgl. Lipowsky 2010). In einem zweiten Schritt wurden Gespräche mit verschiedenen Akteur*innen der Fortund Weiterbildungspraxis geführt (Schulleitungen, Akademieleitungen, Verantwortliche im Ministerium und in Regierungspräsidien, Leitung ministeriumsnaher Institute zur Qualitätssicherung an Schulen) um Informationen über die Organisationsstrukturen und die Angebotsentwicklung zu gewinnen. Eine zentrale Erkenntnis daraus war »das weitgehende Fehlen wissenschaftlicher [...] Expertise in den Angeboten « (Schmidt-Hertha und Werner 2019, 255). Der dritte Schritt diente dazu, die Fortbildungsbedarfe der Lehrkräfte zu eruieren. Da keine eigene Bedarfsanalyse durchgeführt werden konnte, wurde auf bereits vorhandene Studien zurückgegriffen. Zunächst zeigte sich der bildungspolitische und gesellschaftliche Bedarf an wissenschaftlich fundierten Fortbildungsangeboten zum professionellen Umgang mit heterogenen Lerngruppen bereits durch die Projektfinanzierung durch das BMBF. Auch bei den Lehrkräften wurde dieser Bedarf deutlich: Die Lehrkräfte fühlen sich bezüglich der Durchführung inklusiven Unterrichts überfordert, verunsichert und nur unzureichend qualifiziert, sodass ein hoher Bedarf an Fortbildungsangeboten zu diesem Thema existiert (vgl. Leipziger, Tretter und Gebhard 2012; Amrhein 2011; Hüfner 2012; Terhart 2015). Über die Feld- und Bedarfsanalyse hinaus wurden die Akteur*innen der Praxis kontinuierlich in den Entwicklungsprozess des Fortbildungskonzeptes miteinbezogen, wie im Folgenden dargestellt wird.

\subsubsection{Theoretische Perspektive}

\section{Heterogenitätssensibles Lehrhandeln}

Die Heterogenität der Schüler*innen wird oftmals als eine von außen in den Unterricht hineingetragene Tatsache dargestellt, und im Professionalisierungsdiskurs entsprechend unter der Frage verhandelt, welche einzelnen Kompetenzen Lehrkräfte für den richtigen Umgang mit den Unterschieden ihrer Schüler*innen erlernen müssen. Dabei wird außer Acht gelassen, dass >Heterogenität $<$ immer auch ein von Beobachtung abhängiges Phänomen ist, $\gg$ das durch institutionelle und professionelle Unterscheidungspraxen schul- und unterrichtsintern generiert wird « (Emmerich und Goldmann 2018). Um im Schulalltag kommunizieren und handeln zu können, müssen Lehrkräfte Unterscheidungen vornehmen, diesen pädagogische Relevanz zuweisen und sie zur Graduierung von Leistung heranziehen (vgl. Emmerich und Hormel 2013). Heterogenitätssensibles 
Lehrhandeln zeichnet sich dadurch aus, dass die Lehrkräfte sich dieser von der eigenen Beobachtung abhängigen Differenzierungspraxis bewusst sind und in der Lage dazu sind, diese kontinuierlich zu reflektieren und ggf. weiterzuentwickeln (vgl. Idel, Rabenstein und Ricken 2017). Dabei ist die Frage zentral, »wie und auf Basis welcher personenbezogenen Unterscheidungen Lehrkräfte Heterogenität zu einem für sie relevanten Sachverhalt machen und welche Unterrichtsprobleme damit möglicherweise selbst erzeugt werden « (Emmerich und Goldmann 2018). Die komplexe, oftmals widersprüchliche Unterrichtswirklichkeit erfordert dabei von Lehrenden die Fähigkeit, Theorie und Praxis immer wieder neu produktiv miteinander zu verbinden und situativ angemessen zu handeln (vgl. Tietgens 1988; Helsper 2016).

Da Lehrkräfte in der Lehrsituation selbst ihre Beobachtungspraxis nicht reflektieren können, ist eine distanzierte Betrachtung der Situation notwendig. Gemäß der Systemtheorie Luhmanns kann hier zwischen einer Beobachtung erster und einer Beobachtung zweiter Ordnung unterschieden werden. Während die Beobachtung erster Ordnung die Beobachtungspraxis selbst meint, umfasst die Beobachtung zweiter Ordnung die Einnahme einer Analyseperspektive auf eben diese Beobachtungspraxis. Die Beobachtung zweiter Ordnung ist notwendig, um herauszufinden, wie und mit welchen Folgen ein/e Beobachter*in die Realität konstruiert und auch, »welche alternativen Unterscheidungen und Situationsdeutungen in der jeweiligen Situation möglich gewesen wären « (Emmerich und Goldmann 2018; vgl. u. a. Siebert 2011; Emmerich und Hormel 2013). Um das heterogenitätssensible Lehrhandeln der Lehrkräfte zu fördern, ist es im Rahmen von Professionalisierungsprozessen notwendig, die Lehrkräfte für die Bedeutung ihrer Beobachtungs- und Handlungspraxis zu sensibilisieren und sie dabei zu unterstützen, eine Analyseperspektive auf diese Beobachtungs- und Handlungspraxis einzunehmen (Beobachtung zweiter Ordnung). Diese reflexive Auseinandersetzung mit der eigenen Beobachtungs- und Handlungspraxis im Unterricht ist Voraussetzung dafür, dass die Lehrkräfte alternative Beobachtungen und Handlungen ableiten können (vgl. Siebert 2011; Emmerich und Goldmann 2018). Die Lehrer*innen müssen also zunächst ein Bewusstsein für das eigenen Beobachten und Handeln entwickeln, bevor sie es dann ggf. anpassen und weiterentwickeln können.

\section{Reflexionszentrierte Lernumgebungen}

Das zugrunde gelegte Professionalisierungsverständnis zum Umgang mit heterogenen Lerngruppen zielt auf die Herausbildung eines (selbst-)reflexiven, wissenschaftlichen Habitus (vgl. Helsper 2016). Dabei kommt der ergebnisorientierten Problem- bzw. (Selbst-)Reflexion ein zentraler Stellenwert zu. Diese beschreibt »einen bewussten Prozess, bei dem eine Person ihre Vorstellungen oder Handlungen durchdenkt und expliziert, die sich auf ihr reales und ideales Selbstkonzept beziehen. Ergebnisorientiert ist die Selbstreflexion, wenn die Person dabei Folgerungen für künftige Handlungen 
oder Selbstreflexionen entwickelt « (Greif 2008, 40, zur Bedeutung für Professionalisierungsprozesse vgl. auch Pachner 2013, 2014). Die Herausbildung eines reflexiven Habitus erfolgt in der Auseinandersetzung mit Fällen aus der Unterrichtspraxis, die es den Lehrkräften ermöglicht, handlungsentlastet verschiedene Situationen zu reflektieren und zu analysieren (vgl. Helsper 2016). Um sich seiner eigenen Perspektive bewusst zu werden und um andere Perspektiven von berufstätigen Lehrkräften kennenzulernen sind außerdem im direkten Austausch stattfindende Perspektivverschränkungen notwendig (vgl. Siebert 2012). Damit das Gelernte auch in die Praxis übertragen wird, muss weiter der Praxistransfer bereits in der Fortbildung angebahnt werden (vgl. Ho, Watkins und Kelly 2001). Die (Selbst-)Reflexion, die Fallarbeit, die im Austausch stattfindenden Perspektivverschränkungen und der Praxistransfer sind zentrale Elemente transformativen Lernens (vgl. Ho, Watkins und Kelly 2001, Mezirow 1997), die in reflexionszentrierte Lernumgebungen besonders gut integriert werden können bzw. bereits Bestandteile dieser sind (vgl. Hof 2007). Reflexionszentrierte Lernumgebungen zeichnen sich dadurch aus, dass Wissen nicht in erster Linie von den Dozierenden »vermittelt «, sondern von den Teilnehmenden selbst anhand konkreter Fälle aus der Praxis eingebracht und erarbeitet wird (vgl. Hof 2007). Die Lehrkräfte werden dabei als erfahrene Expert*innen für den Umgang mit heterogenen Lerngruppen adressiert (vgl. Siebert 2007; Helsper 2016). Die Dozierenden fungieren als Moderator*innen und Berater*innen, die Lehr-/Lernprozesse arrangieren und die Lehrkräfte bei der Einnahme der Analyseperspektive (im Sinne einer Beobachtung zweiter Ordnung) und der Entwicklung von Beobachtungs- und Handlungsalternativen unterstützen (vgl. Siebert 2007). Das Fortbildungsangebot wurde auf Basis dieser theoretischen Grundlagen sowie unter Einbezug struktureller, didaktischer und prozessbezogener Merkmale wirksamer Fortbildungsangebote (vgl. Lipowsky 2009) entwickelt.

Die praktische und die theoretische Perspektive auf die Professionalisierung von Lehrkräften zum Umgang mit heterogenen Lerngruppen bildeten die Grundlage für die Gestaltung eines Fortbildungs-Prototyps, dessen Entwicklung im Folgenden dargestellt wird. Im Rahmen der Evaluation wurde dabei zum einen die Annahme überprüft, dass das Fortbildungskonzept den Bedarfen der Lehrkräfte gerecht wird. Dies umfasst, dass die Lehrkräfte das Angebot als relevant wahrnehmen, an diesem aktiv teilnehmen und dass sie zufrieden mit diesem sind. Zum anderen wurde angenommen, dass das Fortbildungskonzept heterogenitätssensibles Lehrhandeln fördern kann. Dabei zeigt sich heterogenitätssensibles Lehrhandeln in einem Bewusstsein für die eigene Beobachtungs- und Handlungspraxis, welches sich auch im Handeln der Lehrkräfte niederschlagen sollte.

Die zentrale Fragestellung lautete dementsprechend: Inwiefern kann ein Bewusstsein der Teilnehmenden für die eigene Beobachtungs- und Handlungspraxis geschaffen werden und inwiefern geht das mit einer Veränderung dieser Praxis einher? 


\subsection{Entwicklung der Fortbildung im Austausch mit Expert*innen aus Wissenschaft und Praxis}

Nachdem Probleme und Ziele präzisiert und der theoretische Bezugsrahmen erläutert wurde, folgen gemäß dem Prozessmodell des Design-Based Research Ansatzes die Entwicklung, Erprobung und Evaluation der Veranstaltung (vgl. Euler 2014b). Diese drei Schritte wurden im Rahmen der vorliegenden Studie zweimal durchlaufen (Abschnitt 2.2-2.5). Der Prototyp des Fortbildungskonzeptes wurde in einem interdisziplinären Team aus Erwachsenenbildung und Allgemeiner Pädagogik mit Schwerpunkt Inklusion, Heterogenität, Diversität auf Basis der dargelegten praktischen und wissenschaftlichen Befunde entwickelt. Mit dem Ziel, das Konzept vor dem Praxistest zu verfeinern, wurde es mit Expert*innen aus Wissenschaft und Praxis im Rahmen eines »Round Tables « diskutiert. Dabei war es ein zentrales Anliegen, unterschiedliche Perspektiven auf die praktische Umsetzbarkeit und die Attraktivität bzw. den Mehrwert und Nutzen des Konzeptes zu gewinnen (vgl. Euler 2014b). Von den Expert*innen wurde resümiert, dass das Konzept innovativ, methodisch-didaktisch stimmig und nachhaltig sei, gleichzeitig aber auch sehr herausfordernd für die berufstätigen Lehrkräfte. So müsse der Gewinn des Konzeptes für die Lehrkräfte, den die Expert*innen in einer intensiven Auseinandersetzung mit und Arbeit am eigenen Lehrhandeln sehen, bereits in der Bewerbung des Angebotes vermittelt werden. Ansonsten sei es schwierig, die Lehrkräfte für eine Teilnahme an einem über drei Monate lang andauernden Fortbildungsangebot zu gewinnen. Die im Rahmen dieses Round Tables gewonnenen Erkenntnisse wurden im Fortbildungsteam diskutiert und in das Fortbildungskonzept eingearbeitet.

\section{Das Fortbildungskonzept ${ }^{3}$}

Die Fortbildungsreihe »Professioneller Umgang mit heterogenen Lerngruppen - das eigene Lehrhandeln analysieren, fördern und weiterentwickeln « richtet sich an Lehrkräfte der Sekundarstufen und umfasst drei Präsenztermine und zwei online begleitete Anwendungsphasen.

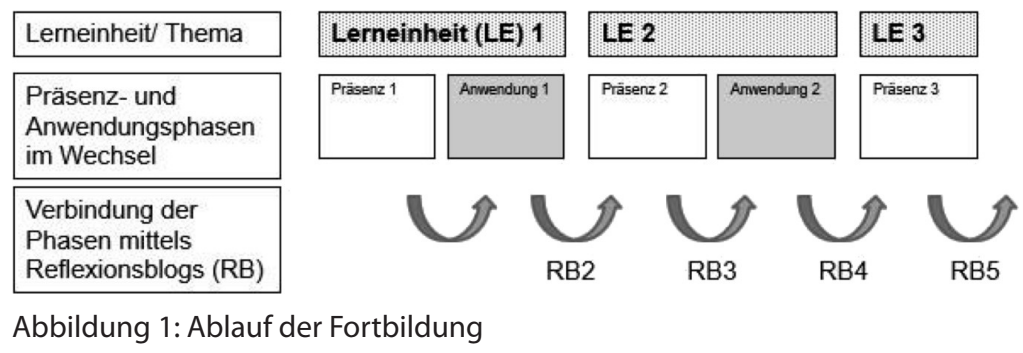


In der ersten Präsenzsitzung findet eine Hinführung zum Thema Heterogenität statt, in der Heterogenität nach Emmerich und Goldmann (2018) als ein von Beobachtung abhängiger Sachverhalt eingeführt wird (siehe Abschnitt 2.2). Daraufhin wird gemeinsam im Plenum die Einnahme einer Analyseperspektive erprobt, indem auf Basis fremder Fälle die Differenzierungspraxis (vgl. Emmerich und Hormel 2013; Idel, Rabenstein und Ricken 2017) der darin agierenden Lehrkräfte nachvollzogen werden soll. Die (Selbst-)Reflexion der eigenen Beobachtungs- und Handlungspraxis wird mithilfe sogenannter Reflexionsblogs (RB) angeregt. Dabei werden die Teilnehmenden nach der ersten Präsenzsitzung zunächst dazu eingeladen, eigene Fälle aus ihrer Unterrichtspraxis zu erinnern und zu verschriftlichen, um auf diese Weise die eigene Beobachtungs- und Handlungspraxis im Unterricht explizit und damit reflektierbar zu machen. Anschließend sollen die Teilnehmenden ihre eigenen Situationsbeschreibungen daraufhin analysieren, welche alternativen Beobachtungsweisen, Deutungen und Handlungen in der jeweiligen Situation auch möglich gewesen wären (RB2) (vgl. Emmerich und Goldmann 2018; Arnold 1985). In den Präsenzsitzungen (P) werden diese Fälle mit den anderen Teilnehmenden analysiert und diskutiert, sodass neue Perspektiven auf die eigene Unterrichtspraxis eingenommen und Beobachtungs- und Handlungsalternativen kennengelernt werden können (P2 und P3). In RB3 sollen die Teilnehmenden dann etwa für herausfordernde Unterrichtssituationen alternative Handlungs- und/oder Beobachtungsweisen entwickeln. In der darauffolgenden zweiten Anwendungsphase erproben sie diese Beobachtungs- und Handlungsalternativen in ihrer Unterrichtspraxis. Anschließend sollen die Teilnehmenden anhand einer weiteren Situationsbeschreibung reflektieren, wie sie das neue Beobachten und Handeln und seine Umsetzung bewerten, was sie beibehalten und was sie ändern möchten (RB4). In RB5 reflektieren die Teilnehmenden anhand der vorausgegangen Reflexionsblogs ihre Entwicklung über die gesamte Fortbildung hinweg.

\subsection{Erprobung und Evaluation des Fortbildungskonzeptes Runde 1}

Ziel der Evaluationsstudie war es, die eingangs getroffenen Annahmen zu prüfen und das Konzept schrittweise zu verbessern. Dazu eignet sich eine formative Evaluation, in die die Lehrkräfte als zentrale Akteur*innen direkt einbezogen werden, in besonderem Maße (vgl. Euler 2014b). Die Evaluation des Angebots fand dabei sowohl auf der Ebene der Zufriedenheit als auch auf der Ebene der Lernprozesse der Teilnehmenden im Rahmen der Fortbildung statt (vgl. Lipowsky 2009). Um die beiden Fragestellungen beantworten zu können wurden folgende Zugänge gewählt. 


\subsubsection{Evaluation der Zufriedenheit der Teilnehmenden mit dem Fortbildungskonzept}

\section{Forschungsdesign}

Um herauszufinden, ob das Fortbildungskonzept den Bedarfen der Lehrkräfte entspricht, wurde die Zufriedenheit der Teilnehmer*innen mit dem Konzept im Fortbildungsverlauf kontinuierlich erhoben. Dazu wurden die drei schriftlich fixierten Beobachtungsprotokolle, die im Rahmen der Teilnehmenden Beobachtung (TB 1-3) in den Präsenzsitzungen angefertigt wurden, herangezogen. In diesen liegt das Feedback der Teilnehmenden am Ende jeder Präsenzsitzung sowie im Rahmen der qualitativen Abschlussevaluation vor. Zusätzlich wurden die Ergebnisse der »Bilanzwaage « ${ }^{4}$, die die Teilnehmenden im Rahmen der Abschlussevaluation angefertigt hatten, sowie die E-Mails der Teilnehmenden, in denen zum Teil sehr ausführliches Feedback zum Konzept enthalten ist, zur Auswertung hinzugezogen.

In der ersten Fortbildungsrunde waren 17 Lehrkräfte ${ }^{5}$ beteiligt, die alle in die Auswertung zur Zufriedenheit mit dem Fortbildungskonzept einbezogen wurden, auch wenn sie zum Beispiel nur bei der ersten Präsenzsitzung dabei waren. Die Daten wurden mithilfe der Qualitativen Inhaltsanalyse (vgl. Mayring 2015) ausgewertet.

\section{Ergebnisse}

Die Frage, ob das reflexionszentrierte Fortbildungsangebot den Bedarfen der Lehrkräfte entspricht, kann anhand des qualitativen Teilnehmerfeedbacks beantwortet werden. Bereits die hohen Anmeldezahlen bestätigten den Bedarf der Lehrkräfte nach Fortbildungen zum Thema » professioneller Umgang mit heterogenen Lerngruppen «. Während der einzelnen Phasen war die Teilnahme relativ stabil, auch wenn nicht alle Teilnehmenden durchgehend aktiv an den Präsenz- und Anwendungsphasen beteiligt waren. Im Bewusstsein darüber, dass es sich um ein sehr herausforderndes und zeitintensives Konzept handelt (siehe auch Ergebnisse des Round Tables in 2.2), sind diese Zahlen als sehr positiv zu bewerten. Dabei kann insgesamt eine hohe Zufriedenheit mit dem Fortbildungskonzept verzeichnet werden. Als besonders lernförderlich wurden von den Teilnehmenden der Austausch und die Beratungselemente hervorgehoben und auch der Reflexionsarbeit wurde ein bedeutender Stellenwert für die eigene Entwicklung zugeschrieben (TB3, Z. 182f. und Bilanzwaage). So regte die Fortbildung die Teilnehmenden zum selbstkritischen Reflektieren, zur Einnahme neuer Perspektiven (auch, was den Begriff »Heterogenität « betrifft) und zur Selbsterkenntnis an (vgl. TB1, Z. 6ff.). Dabei wurde das Gezwungen-sein, über das eigene Handeln zu reflektieren als herausfordernd aber gleichzeitig auch ertragreich erlebt:

»Nach der ersten Sitzung war ich erschlagen, es ist halt Uni. Wusste nicht, kriege ich das hin? Es wurde aber dann konkret. Man muss Hürden überwinden, gezwungen werden, 
darüber nachzudenken, was man tut. Für mich war das sehr bereichernd. Diese Runde heute hat es komplett in die Praxis geführt, der rote Faden der Veranstaltung war dann gut nachvollziehbar. So soll ein Erkenntnisprozess sein « (TB3, Z. 185).

Positiv erwähnt wurden außerdem der Input und das hohe inhaltliche Niveau, wobei die » sozialwissenschaftliche Sprache « auch einzelnen Teilnehmenden das Verständnis der Fortbildungsinhalte erschwerte (vgl. E-Mail TN1). Bezüglich des wissenschaftlichen Zugangs zum Thema und der universitären Verortung resümierte ein/e Teilnehmer*in:

»Ich fand es total erfrischend, dass es universitär war, dass man in die Theorie geschaut hat und nicht auf der Oberfläche geblieben ist. Nicht so wie in anderen Fobis da wird gesagt, was man im Unterricht machen soll und man bekommt ein Handbuch dafür. Da fühle ich mich nicht für voll genommen, das ist so aufgezwungen « (TB3, Z. 186).

Kritisch bewertet wurde von einigen, dass zu Beginn der Fortbildung der Ansatz des Konzeptes bzw. das Vorgehen und die Ziele nicht klar waren und die Teilnehmenden nicht wussten, worauf das Ganze hinauslaufen sollte (vgl. TB3, Z. 183f.). Glücklicherweise hielt dies jedoch keinen davon ab, die Fortbildung weiter zu besuchen. Nur ein/e Teilnehmer*in meldete sich nach der ersten Präsenzsitzung ab, weil sie/er mit nicht erfüllbaren Erwartungen in die Fortbildung gekommen war: Sie/Er hatte gehofft, »Handwerkszeug « im Sinne konkreter Methoden und Modelle zum professionellen Umgang mit Heterogenität zu erlangen (vgl. E-Mail TN17). Diese Erwartungen hatten zwar auch andere Teilnehmende, diese ließen sich aber trotzdem auf das Fortbildungskonzept ein und profitierten letztendlich von der Teilnahme. Bemängelt wurden die nicht ganz intuitive Bedienung der Lernplattform sowie die zu kurzen Anwendungsphasen, in denen die Arbeitsaufgaben kaum bewältigt werden konnten (vgl. Bilanzwaage).

Das Feedback der Teilnehmenden im Fortbildungsverlauf wurde schon in der ersten Durchführungsrunde dafür genutzt, das Angebot kontinuierlich weiter zu entwickeln. Beispielsweise wurde das konkrete Vorgehen und der methodisch-didaktische Ansatz des Konzeptes auf das Feedback der Teilnehmenden hin zu Beginn der zweiten Präsenzsitzung noch einmal detaillierter erläutert. Außerdem wurde deutlicher begründet, weshalb es uns nicht sinnvoll erscheint, »Rezeptwissen « im Sinne von Methoden und Modellen für den Umgang mit heterogenen Lerngruppen zu vermitteln. Darüber hinaus wurde versucht, die wissenschaftlichen Inhalte bzw. Sprache noch stärker herunter zu brechen bzw. Fachbegriffe noch öfter zu erklären. Gleichzeitig fand eine engmaschige Betreuung der Teilnehmenden während der Anwendungsphasen statt, sodass auch dort Fragen aller Art geklärt werden konnten. Die Punkte, die wir im Rahmen der ersten Fortbildungsrunde nicht direkt verändern konnten, wie zum Beispiel die Lernplattform oder die Dauer der Anwendungsphasen wurden in der zweiten Runde berücksichtigt. 


\subsubsection{Evaluation der Lernprozesse der Teilnehmenden im Fortbildungsverlauf}

\section{Forschungsdesign}

Um herauszufinden, inwiefern ein Bewusstsein für die eigene Beobachtungs- und Handlungspraxis im Umgang mit Heterogenität geschaffen werden konnte und inwiefern das auch mit einer Veränderung dieser Praxis einhergeht, wurde unterschiedliches, qualitatives Datenmaterial trianguliert (vgl. Flick 2009). Dies waren zum einen die schriftlich fixierten Beobachtungsprotokolle aus den drei Präsenzsitzungen und zum anderen die Reflexionsblogs der Teilnehmenden. Während die Beobachtungsprotokolle direkten Einblick in die Fortbildungspraxis und den dort stattfindenden Austausch ermöglichen, wurde mithilfe der Reflexionsblogs die Selbstreflexion der Teilnehmenden erfasst. Es wurden diejenigen Teilnehmenden in die Stichprobe einbezogen, die aktiv an allen Präsenzterminen und Anwendungsphasen beteiligt waren, da dies die Voraussetzung für die Auseinandersetzung mit der eigenen Beobachtungs- und Handlungspraxis ist. Für die sieben ausgewählten Lehrkräfte ${ }^{6}$ liegen sowohl Daten in den Beobachtungsprotokollen als auch in den individuellen Reflexionsblogs vor. Um die Entwicklungsprozesse der Lehrkräfte im Fortbildungsverlauf sichtbar zu machen, wurden die Beobachtungsprotokolle und die Reflexionsblogs mittels Qualitativer Inhaltsanalyse (Mayring 2015) ausgewertet.

\section{Ergebnisse}

Die beiden aus der Theorie deduktiv abgeleiteten Hauptkategorien, die Bewusstheit der eigenen Beobachtungs-und Handlungspraxis sowie die Bereitschaft, das eigene Beobachten und Handeln weiterzuentwickeln, konnten im Material vorgefunden und kodiert werden. Im Zuge einer induktiven Kategorienbildung wurden weiter verschiedene Ausprägungen dieser beiden Hauptkategorien festgestellt. Abbildung 2 zeigt die verschiedenen Unterkategorien der Bewusstheit und der Bereitschaft sowie die Anzahl der Teilnehmenden, bei denen diese im Rahmen der jeweiligen Reflexionsphase sichtbar wurden.

Es zeigt sich dabei, dass im Fortbildungsverlauf die Anzahl der Teilnehmenden zunimmt, die die jeweiligen Ausprägungen aufweisen, weshalb von einer (Weiter-)Entwicklung des heterogenitätssensiblen Lehrhandelns der Teilnehmenden gesprochen werden kann.

\section{Die Entwicklung der Bewusstheit der eigenen Beobachtungs- und Handlungspraxis}

Die unterschiedlichen Ausprägungen der Bewusstheit der eigenen Beobachtungs- und Handlungspraxis der Teilnehmenden konnten anhand der Selbstreflexionen in den Reflexionsblogs 2, 3 und 5 herausgearbeitet werden. Die Kategorie »Ist sich bewusst, dass er/sie differenziert « beschreibt, dass die Lehrkräfte wissen, dass sie selbst Unterschiede zwischen den Schüler*innen erzeugen und diese nicht einfach naturgegeben sind. 


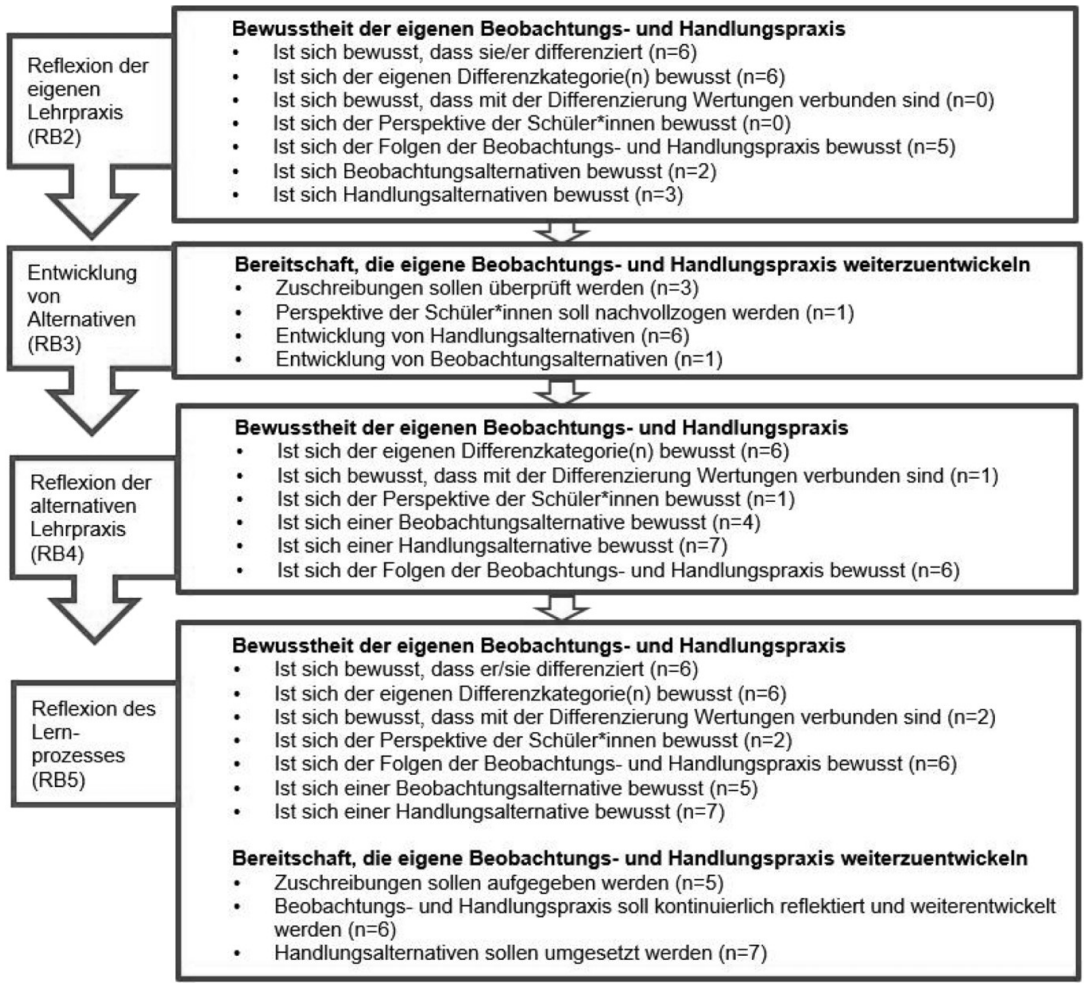

Abbildung 2: Ergebnisse der Qualitativen Inhaltsanalyse der Fortbildungsrunde 1 $(n=7)$ (eigene Darstellung).

»Ich merke, wie ich sie kategorisiere, wie ich ihnen Eigenschaften zuschreibe aufgrund von Vorerfahrungen, die ich mit ihnen gemacht habe, und dann ihre Handlungen im Unterricht unterschiedlich bewerte ( $>$ Hurra, sie meldet sich $<$, $>$ Mist, sie ruft schon wieder rein $<$ etc. $) \ll(T N 3, R B 2, Z .16)$.

Eine weitere Ausprägung beschreibt, dass die Teilnehmenden die Differenzkategorien benennen können, die ihrem Beobachten und Handeln zugrunde liegen:

$\gg$ Ich habe einen Schüler als > sehr langsam< abgespeichert. Ich habe mich verstärkt um diesen Schüler gekümmert « (TN7, RB2, Z.12).

Weiter wird auch das Bewusstsein über die Folgen der eigenen Beobachtungs- und Handlungspraxis als Ausprägung definiert. So ist sich eine Teilnehmerin bewusst darüber, dass 
sie durch ihr Verhalten einem Flüchtlingskind besonders viel Aufmerksamkeit schenkt und die anderen dadurch hintenanstellt (vgl. TN 6, RB2, 9ff.). Das Bewusstsein für Beobachtungsalternativen und das Bewusstsein für Handlungsalternativen sind zwei weitere Ausprägungen, die in der Reflexion zutage treten. Auch lassen sich ein Bewusstsein darüber, dass die eigene Differenzierungspraxis mit wertenden Zuschreibungen einhergeht (z. B. eine Differenzierung nach guten und schlechten Schüler*innen) sowie ein Bewusstsein über die Perspektive der Schüler*innen seitens der Teilnehmenden erkennen. Diese beiden Ausprägungen werden jedoch erst ab dem Reflexionsblog 4 sichtbar.

\section{Die Bereitschaft, das eigene Beobachten und Handeln weiterzuentwickeln und Alternativen in die eigene Unterrichtspraxis umzusetzen}

Die Ausprägungen der Bereitschaft, das eigene Beobachten und Handeln weiterzuentwickeln zeigen sich im Rahmen der Planung des alternativen Beobachtens und Handelns in Reflexionsblog 3. Auch werden diese im Rahmen der Reflexion der eigenen Lernentwicklung in Reflexionsblog 5 sichtbar. Dabei treten folgende Ausprägungen auf: Die Teilnehmenden wollen beispielsweise die Zuschreibungen, die sie gegenüber den Schüler*innen vornehmen, überprüfen (RB3) bzw. diese, wenn sie sich in der Überprüfung entweder als falsch oder als der Förderung der Schüler*innen nicht dienlich erwiesen haben, auch aufgeben (RB5).

»Im Laufe der Fortbildung hat sich meine Beobachtung dahingehend geändert, dass auch andere SuS [Anm.d. V.: Schülerinnen und Schüler] motiviert und gut sind, die vielleicht weniger extrovertiert sind und auch Aufmerksamkeit verdienen. Ich versuche individueller und stundenspezifischer zu beurteilen wer gut/schlecht, motiviert/unmotiviert, hilfebedürftig ist « (TN12, RB5, Z. 54).

Die Teilnehmenden planen in Reflexionsblog 3 außerdem, dass sie Handlungsalternativen in der Praxis erproben, die Perspektive der Schüler*innen nachvollziehen oder Situationen einmal anders beobachten möchten:

»Wer braucht wirklich (mehr) Aufmerksamkeit und wer nicht? Was sind wirkliche Störungen und was die Suche nach Aufmerksamkeit? (TN12, RB3, Z.48)

In der Reflexion des Lernprozesses (RB5) resümieren die Teilnehmenden außerdem, dass sie die Handlungsalternativen weiterhin beibehalten möchten sowie dass sie ihre Beobachtungs- und Handlungspraxis nun kontinuierlich reflektieren und weiterentwickeln (möchten):

»Ich versuche, sie immer mal wieder $>$ neutral< anzuschauen, mit $>$ mal sehen, wie ist es heute?< Das geht nicht durchgehend, aber immer mal wieder, situationsweise. Dabei kam 
mein Blick auch auf Entwicklungen, die die SuS gemacht haben. Ich denke nicht immer $>$ jemand ist so<, sondern: >interessant, heute ist das so <«(TN4, RB5, Z. 49).

\subsubsection{Interpretation der Ergebnisse}

Die relativ stabile Teilnahme und das überwiegend positive Feedback der Teilnehmenden können als Indikatoren dafür gesehen werden, dass das Konzept auf die Bedarfe der Lehrkräfte reagiert. Die Ergebnisse weisen auf eine hohe Zufriedenheit der Teilnehmenden mit dem Fortbildungskonzept hin, obwohl ihnen der neue Ansatz zu Beginn wenig eingänglich und auch verwirrend erscheint. Es wird deutlich, dass es seitens der Lehrkräfte eines »sich-Einlassens « auf das Konzept bedarf, das von traditionellen, wissenszentrierten Lernumgebungen (vgl. Hof 2007) stark abweicht: Es wird kein konkretes Wissen und Handwerkszeug vermittelt, sondern die Lehrkräfte müssen selbst tätig werden, sich Wissen erarbeiten und mit Unsicherheiten umgehen. So resümierte ein/e Teilnehmer*in am Ende der Veranstaltung:

»Aber Sie haben ja am Anfang gesagt, dass sie uns nicht zeigen, wie es geht. Vielleicht geht das Lernen ja auch nur über die Unsicherheit « (TB3, Z. 192).

Das Konzept erfordert also von den Teilnehmenden ein hohes Engagement, eine Offenheit für Neues sowie Veränderungsbereitschaft. Lassen sich die Teilnehmenden auf das Konzept ein und nehmen aktiv teil, dann können tatsächlich ein Bewusstsein für die eigene Beobachtungs- und Handlungspraxis im Umgang mit Heterogenität geschaffen werden und Veränderungen stattfinden (vgl. auch Baust und Pachner 2020). Die aus dem Material heraus gebildeten, induktiven Kategorien zeigen, was im Rahmen dieser Prozesse bei den einzelnen Teilnehmenden passiert. So lassen sich unterschiedliche Ausprägungen der Bewusstheit der eigenen Beobachtungs- und Handlungspraxis im Umgang mit Heterogenität finden. Im Laufe der Fortbildung ist diesbezüglich eine Ausdifferenzierung zu beobachten, weshalb resümiert werden kann, dass eine Weiterentwicklung der Teilnehmenden stattgefunden hat. Gleichzeitig lassen sich unterschiedliche Absichten, die eigene Beobachtungs- und Handlungspraxis weiterzuentwickeln und Alternativen in der eigenen Unterrichtspraxis umzusetzen, finden. Fast alle Teilnehmenden resümieren am Ende der Veranstaltung, dass sie ihre Beobachtungs- und Handlungspraxis kontinuierlich überprüfen und weiterentwickeln möchten, was auf die Einnahme eines » reflexiven Habitus « (vgl. Helsper 2016) hindeuten könnte. Die Ergebnisse zeigen weiter, dass das reflexionszentrierte Fortbildungskonzept eine Auseinandersetzung und auch Veränderung der individuellen Beobachtungs- und Handlungspraxis ermöglichen kann (vgl. Siebert 2012). Die Anregung zur ergebnisorientierten (Selbst-)Reflexion (Greif 2008, Pachner 2013, 2014), 
die Fallarbeit (vgl. Helsper 2016), der auf Perspektivverschränkung angelegte Austausch (vgl. Siebert 2012) sowie die integrierten Erprobungsphasen wurden von den Teilnehmenden als Anlässe dafür genutzt, sich neuer Aspekte ihrer Beobachtungsund Handlungspraxis bewusst zu werden und diese entsprechend weiterzuentwickeln (vgl. Hof 2007).

\subsection{Erste Handlungsempfehlungen und Re-Design}

Auf Basis der Evaluationsergebnisse konnten vorläufige Handlungsempfehlungen für die Gestaltung reflexionszentrierter Fortbildungsformate zum professionellen Umgang mit heterogenen Lerngruppen im universitären Kontext (vgl. Euler 2014b) abgeleitet werden. Es haben sich folgende Elemente des Fortbildungskonzeptes als förderlich für die Lernentwicklung der Teilnehmenden erwiesen: Die gezielte Anregung zur handlungsnahen Reflexion auf Basis der von den Teilnehmenden eingebrachten Fälle, die Verbindung von Problem- und Selbstreflexion mit dem Austausch der Teilnehmenden in den Präsenzsitzungen sowie die online betreuten Anwendungsphasen. Die handlungsnahe und ergebnisorientierte (Selbst-)Reflexion (vgl. Greif 2008, Pachner 2013, 2014) wurde durch die Arbeit mit Fällen aus der eigenen Unterrichtspraxis der Teilnehmenden ermöglicht (vgl. Helsper 2016). Die Ergebnisse der (Selbst-)Reflexion waren wiederum die Grundlage für die Fallarbeit im erfahrungsbasierten Austausch, bei dem an bereits bestehendes Wissen der Lehrkräfte angeknüpft wurde und die Teilnehmenden ihre Perspektiven verschränken und erweitern konnten (vgl. Siebert 2007). Dabei kam auch den beiden online betreuten Anwendungsphasen eine zentrale Bedeutung zu, ohne die die Analyse und Erprobung von Beobachtungs- und Handlungsalternativen nicht hätte stattfinden können und die den Transfer der Inhalte in die Unterrichtspraxis ermöglichten (vgl. Ho, Watkins und Kelly 2001).

Das Fortbildungskonzept, das Evaluationsdesign sowie die Lernziele wurden im zweiten Fortbildungsdurchlauf beibehalten. Bezüglich der Durchführung wurden folgende Punkte angepasst bzw. verändert: Es wurde zum einen eine andere Lernplattform eingesetzt, die eine leichtere Bedienung ermöglicht, und zum anderen wurde eine längere Dauer für die Anwendungsphasen angesetzt, damit die Lehrkräfte genügend Zeit dazu haben, Fälle aus ihrer Unterrichtspraxis zu beschreiben und zu analysieren sowie Alternativen in der Praxis zu erproben. Um sie darüber hinaus dazu anzuregen, die Aufgaben der Anwendungsphase vollständig zu bearbeiten, wurden die einzelnen Aufgabenteile nicht auf einmal gestellt, sondern die Teilnehmenden erhielten kleinere, zeitlich versetzte Arbeitsaufträge sowie regelmäßige Erinnerungen in Form von $\gg$ Remindern «. Außerdem wurden der Ansatz des Konzeptes sowie die damit verbundenen fachsprachlichen Grundlagen zu Beginn der zweiten Veranstaltung ausführlich 
vorgestellt und erläutert. Aufgrund mangelnder zeitlicher Ressourcen übernahm der Dozierende des Fachbereichs Allgemeine Pädagogik in der zweiten Fortbildungsrunde nur den einführenden Input zum heterogenitätssensiblen Lehrhandeln. Alle anderen Veranstaltungsteile wurden von den Dozierenden der Erwachsenenbildung übernommen.

\subsection{Erprobung und Evaluation des Fortbildungskonzeptes Runde 2}

\subsubsection{Evaluation der Zufriedenheit der Teilnehmenden mit dem Fortbildungskonzept}

Die Zufriedenheit der Teilnehmenden wurde auf dieselbe Weise, wie in Abschnitt 2.3.1 vorgestellt, erhoben.

\section{Ergebnisse}

Auch in der zweiten Fortbildungsrunde zeigte sich der Bedarf der Lehrkräfte nach solch einem Angebot. Es meldeten sich weit mehr Lehrkräfte an, als die maximale Teilnehmerzahl von 17 Teilnehmenden es zuließ. Die 17 teilnehmenden Lehrkräfte ${ }^{7}$ wurden alle in die Stichprobe zur Evaluation der Zufriedenheit einbezogen, sofern Feedback von ihnen vorhanden ist (z. B. im Rahmen der Präsenzsitzungen oder per E-Mail). Nach der ersten Präsenzsitzung meldeten sich einige der Lehrkräfte von der Fortbildung ab, was diese hauptsächlich damit begründeten, dass sie mit anderen Erwartungen in die Fortbildung gekommen waren: Sie hatten sich erhofft, konkrete Lösungen für ihre Probleme im Umgang mit Heterogenität beispielsweise für den fachspezifischen Umgang mit Leistungsheterogenität vermittelt zu bekommen (vgl. TB1, Z. 164f.). Darüber hinaus wurde auch das auf Reflexion und Austausch fokussierende Fortbildungskonzept infrage gestellt und der Wunsch nach mehr wissenschaftlichem Input geäußert sowie danach, hauptsächlich von den »Profis « (den Dozierenden) Feedback zu bekommen und weniger von den anderen Teilnehmenden (vgl. E-Mail TN 32). Das Feedback der aktiven Teilnehmenden hingegen fiel ähnlich positiv aus wie in Runde 1. Sie bewerteten Austausch und Fallarbeit sowie den Anwendungsbezug der Fortbildung als besonders lernförderlich und waren auch mit den Rahmenbedingungen sehr zufrieden. Die Teilnehmenden resümierten, dass sie durch die Fortbildung eine neue Betrachtungsweise auf Heterogenität gewonnen hätten (vgl. TB3, Z. 69ff.). Bemängelt wurde von dieser Gruppe, dass der Anfangsinput zu dicht und komplex gewesen sei, die Methode des Kennenlernens zu lang und es wurde (mit einem Augenzwinkern) hinzugefügt, dass es schade sei, dass es keine eindeutigen Lösungen im Umgang mit Heterogenität gebe (vgl. TB3, Z. 361ff.). 
Die Frage, inwiefern das reflexionszentrierte Fortbildungsangebot den Bedarfen der Lehrkräfte entspricht, kann für diese Gruppe nicht einheitlich beantwortet werden. So ist es für einen Teil dieser Lehrkräfte zentral, konkrete, fach- und schulartspezifische Methoden und Strategien für den professionellen Umgang mit Heterogenität an die Hand zu bekommen. Von den Dozierenden wird teilweise erwartet, dass diese eine klassische Vermittlerrolle einnehmen und vor allem Expert*innen für innovative Methoden zum Umgang mit Heterogenität seien. Für diese Lehrkräfte ist das Konzept nicht bedarfsgerecht. Gleichzeitig gibt es auch in der zweiten Fortbildungsrunde Lehrkräfte, die von diesem Konzept profitieren und für die es deshalb als bedarfsgerecht eingestuft werden kann.

\subsubsection{Evaluation der Lernprozesse der Teilnehmenden im Fortbildungsverlauf}

Das Forschungsdesign zur Evaluation der Lernprozesse wurde in Abschnitt 2.3.2 bereits vorgestellt.

Für die Evaluation der Lernprozesse wurden wieder nur die Teilnehmenden in die Stichprobe einbezogen, die aktiv an allen Präsenzterminen und Anwendungsphasen beteiligt waren. Für diese drei Lehrkräfte ${ }^{8}$ liegen sowohl Daten in den Beobachtungsprotokollen als auch in den individuellen Reflexionsblogs vor. Für die Auswertung des Datenmaterials wurde das in Runde 1 entwickelte Kategoriensystem zugrunde gelegt und im Sinne des induktiven Vorgehens weiterentwickelt (Mayring 2015).

Ergebnisse: Die Entwicklung der Bewusstheit der eigenen Beobachtungs-und Handlungspraxis sowie der Bereitschaft, das eigene Beobachten und Handeln weiterzuentwickeln In der zweiten Fortbildungsrunde wurden ebenfalls die beiden Hauptkategorien Die Bewusstheit der eigenen Beobachtungs- und Handlungspraxis entwickeln und Die Bereitschaft, das eigene Beobachten und Handeln weiterzuentwickeln sowie die meisten Ausprägungen dazu in den Daten vorgefunden. Auch für die Teilnehmenden in Runde 2 kann über den Fortbildungsverlauf hinweg eine Ausdifferenzierung dieser beiden Kategorien festgestellt werden. Die Teilnehmenden der Runde 2 unterscheiden sich dabei in folgenden Punkten von denen der Runde 1:

In Reflexionsblog 2 weisen die Teilnehmenden der zweiten Runde weniger Ausprägungen der Bewusstheit der eigenen Beobachtungs- und Handlungspraxis auf. Sie sind sich eigener Differenzkategorien, Folgen für die Beobachtungs- und Handlungspraxis sowie Handlungsalternativen bewusst, jedoch keiner Beobachtungsalternativen und auch nicht darüber, dass differenziert wird. Erst im Rahmen des Reflexionsblog 4 nennen sie Beobachtungsalternativen und auch die Perspektive der Schüler*innen ist ihnen dann bewusst. Im Gegensatz zu den Teilnehmenden der Runde 1 lässt sich im gesamten Fortbildungsverlauf bei keinem der Teilnehmenden der Runde 2 ein Bewusstsein dafür 
finden, dass mit der Differenzierung Wertungen verknüpft sind. Hingegen ist zu beobachten, dass die Teilnehmenden der Runde 2 in Reflexionsblog 3 mehr Ausprägungen der Bereitschaft, das eigene Beobachten und Handeln weiterzuentwickeln, aufzeigen: Sie möchten sich zusätzlich zur Entwicklung von Beobachtungs- und Handlungsalternativen, der Überprüfung von Zuschreibungen sowie der Einnahme der Perspektive der Schüler*innen außerdem Feedback von Kolleg*innen einholen und sich ihrer eigenen Differenzkategorien bewusst werden. In Reflexionsblog 5 wollen die Teilnehmenden der Runde 2 ebenfalls ihre Handlungsalternativen in die Praxis umsetzen und ihre Beobachtungs- und Handlungspraxis kontinuierlich reflektieren und weiterentwickeln. So stellte ein/e Teilnehmer*in bezüglich der neuen Beobachtungspraxis fest:

»Wenn man mal reingeschnuppert hat und sensibilisiert wurde, kann man das nicht wieder auf den Stand vorher zurückfahren. Man nimmt diese neue Perspektive mit in den Unterricht $\ll(T B 3, Z .345)$.

Bei der Auswertung der Daten fällt weiter auf, dass die beiden Gruppen sich in der Bearbeitung der Reflexionsblogs sowie ihrem Diskussionsverhalten unterscheiden. In den Reflexionsblogs dominieren bei den Teilnehmenden der Runde 2 Situationsbeschreibungen mit Erläuterungen, die kaum vom Geschehen abstrahieren, die konkrete Beantwortung der Reflexionsfragen bleibt meistens aus. Die Teilnehmenden der Runde 1 hingegen hatten die Reflexionsfragen größtenteils beantwortet. In den ausgewerteten Sitzungsprotokollen zeigt sich dagegen, dass die Teilnehmenden der zweiten Runde im Austausch mehr und vielfältigere Alternativen diskutieren als die Teilnehmenden in der ersten Runde. Dabei sind alle Teilnehmenden aktiv an der Diskussion beteiligt, während in der ersten Runde einzelne Teilnehmende sich kaum in den Austausch einbringen.

\subsubsection{Interpretation der Ergebnisse}

Die Zufriedenheit der Teilnehmenden der Runde 2 mit dem Fortbildungskonzept war durchwachsen. Dies weicht von den Ergebnissen der ersten Runde deutlich ab, in der sich die Teilnehmenden größtenteils sehr zufrieden gezeigt hatten. Eine mögliche Erklärung dafür ist die anders gelagerte Erwartungshaltung der Teilnehmenden der Runde 2, die sich durch eine starke Fokussierung auf wissenszentrierte Lernumgebungen auszeichnet. Gleichzeitig zeigte sich in der zweiten Runde eine geringere Bereitschaft der Lehrkräfte, sich auf das ungewohnte Fortbildungskonzept einzulassen: Einige der Teilnehmenden meldeten sich bereits nach der ersten Präsenzsitzung $\mathrm{ab}$, was bei den Teilnehmenden der ersten Runde nicht der Fall war, obwohl sie zu Beginn ebenfalls etwas skeptisch gegenüber der reflexionszentrierten Lernumgebung 
waren. Ein weiterer Grund für die geringere Teilnehmerzufriedenheit in Runde 2 könnte durch die kurze Anwesenheit des Heterogenitätsexperten bedingt sein: So war der Heterogenitätsexperte nur mit einem relativ kurzen einführenden Inputvortrag an der Fortbildung beteiligt. Dies könnte die Vermutung der Lehrkräfte bestärkt haben, dass das Thema Heterogenität insgesamt zu kurz komme. Auch möglich ist, dass sich durch das kritische Feedback einzelner Teilnehmender am Ende der ersten Präsenzsitzung eine ungünstige Gruppendynamik einstellte, die einige Lehrkräfte in ihrem anfänglichen Zweifel bestärkte (vgl. TB1, Z. 164ff.).

Eine zentrale Erkenntnis der Evaluation der Lernprozesse der Teilnehmenden beider Fortbildungsrunden ist, dass die in Runde 1 induktiv formulierten Ausprägungen der Bewusstheit der eigenen Beobachtungs- und Handlungspraxis sowie der Bereitschaft, diese weiterzuentwickeln, sich auch in Runde 2 bestätigen. Dies weist darauf hin, dass die beiden aktiven Teilnehmergruppen in Runde 1 und 2 ähnliche Lernprozesse im Rahmen der Fortbildung durchlaufen haben und diese durch die Fortbildung angeregt wurden. Auch wenn sich die beiden Gruppen dadurch unterscheiden, dass die jeweiligen Ausprägungen im Rahmen unterschiedlicher Phasen entwickelt wurden, so lassen sich am Ende der beiden Fortbildungsrunden bei beiden Teilnehmergruppen fast die gleichen Ausprägungen zu den beiden Kategorien finden. In beiden Gruppen konnten Hinweise darauf gefunden werden, dass transformatives Lernen im Sinne der (Weiter-)Entwicklung eines » reflexiven Habitus « angeregt werden konnte (vgl. Helsper 2016). Dieser ist wiederum eine zentrale Voraussetzung für heterogenitätssensibles Lehrhandeln (vgl. Emmerich und Goldmann 2018). Dabei muss einschränkend hinzugesagt werden, dass es sich bzgl. der künftigen Unterrichtsgestaltung um Absichtserklärungen der Lehrkräfte handelt. So wurde das alternative Beobachten und Handeln von den meisten Teilnehmenden zwar bereits im Rahmen der Anwendungsphasen in der Unterrichtspraxis erprobt, es wurde jedoch nicht überprüft, wie sich die Teilnehmenden nach der Fortbildung im Unterricht verhalten.

Mit Blick auf die Bearbeitung der Reflexionsblogs sowie das Diskussionsverhalten der Teilnehmenden in den Präsenzphasen lässt sich Folgendes schließen: Während die Teilnehmenden der Runde 2 hauptsächlich vom Austausch und der gemeinsamen Reflexion der Fälle in den Präsenzsitzungen profitierten, nutzten die Teilnehmenden der Runde 1 vermehrt die Reflexionsblogs, um ihr Beobachten und Handeln zu analysieren. Es wäre möglich, dass dies mit persönlichen Neigungen der Teilnehmenden zusammenhängt, lieber für sich allein und in schriftlicher Form oder aber in der Gruppe zu reflektieren. Auch könnte eine geringer ausgeprägte Bereitschaft oder Fähigkeit zur (Selbst-)Reflexion ein Grund für die weniger intensive Auseinandersetzung mit den Reflexionsfragen sein. Darüber hinaus wäre es denkbar, dass die allzu knappe Beantwortung der Reflexionsfragen durch die Teilnehmenden der Runde 2 mit dem kürzeren Einstiegs-Input zum Thema Heterogenität zusammenhängt, das heißt, dass die Teil- 
nehmenden insgesamt zu wenig in das heterogenitätssensible Lehrhandeln und die Einnahme einer Analyseperspektive eingeführt wurden.

\subsection{Gestaltungsprinzipien}

Ein Ziel des Design-Based Research Ansatzes ist es, kontextualisierte Gestaltungsprinzipien abzuleiten (vgl. Raatz 2016). Dabei handelt es sich nicht um »statements of law « (Euler 2014,31), sondern vielmehr um Richtlinien, die im Zusammenhang mit Ziel und Kontext der Intervention genannt werden müssen (vgl. Euler 2014b). Der von Euler (2014b) beschriebene Prozess aus Designentwicklung bzw. -verfeinerung, Designerprobung und der Ableitung von Handlungsempfehlungen konnte im Rahmen der Studie zweimal durchlaufen werden. Es zeigte sich, dass das reflexionszentrierte Fortbildungskonzept grundsätzlich dazu geeignet ist, die Bewusstheit der eigenen Beobachtungs- und Handlungspraxis im Umgang mit heterogenen Lerngruppen sowie die Bereitschaft dazu, diese weiterzuentwickeln, zu fördern. Folgende Gestaltungsprinzipien für reflexionszentrierte Fortbildungen im universitären Kontext, die darauf abzielen, das professionelle Lehrhandeln im Umgang mit heterogenen Lerngruppen zu fördern, können dabei resümiert werden:

Wie bereits in Abschnitt 2.4 ausgeführt wurde, sind die gezielte Anregung zur handlungsnahen Reflexion auf Basis der Fälle der Teilnehmenden, die Verbindung von Problem- und Selbstreflexion mit dem Austausch der Teilnehmenden sowie die Anwendungsphasen Voraussetzung für die Lernprozesse der Teilnehmenden. Um heterogenitätssensibles Lehrhandeln zu fördern, ist außerdem eine theoretische Hinführung zur Konstruktion von Heterogenität und Differenz sowie zur rekonstruktiven Analyseperspektive notwendig. Darüber hinaus bedarf es für die Analyse des eigenen Lehrhandelns der Anleitung durch die Dozierenden, die außerdem dafür zuständig sind, Reflexion und Austausch entsprechend einzubetten und zu moderieren. Aufseiten der teilnehmenden Lehrkräfte werden ein hohes Engagement sowie die Bereitschaft, sich auf dieses Konzept einzulassen, vorausgesetzt, um die intendierten Lehr-Lernziele zu erreichen. Um die Passung zwischen Interessent*innen und Angebot künftig noch zu verbessern, könnte von den Lehrkräften bei Anmeldung beispielsweise ein Motivationsschreiben eingefordert werden, sodass die Erwartungen vorab abgeglichen werden können. Ein weiterer, wichtiger Aspekt ist, dass die Lehrkräfte, die sich aktiv an dem Fortbildungskonzept beteiligen, von diesem in unterschiedlicher Weise profitieren können. Während die Teilnehmenden der ersten Runde einen großen Nutzen aus der Selbstreflexion in den Reflexionsblogs gezogen haben, profitierten die der zweiten Runde eher von den im Austausch stattfindenden Perspektivverschränkungen. Auch wenn alle Elemente des Fortbildungskonzeptes Voraussetzung für transformatives Lernen sind, so können abhängig von der Teilnehmergruppe also durchaus unterschiedliche Schwerpunkte gesetzt werden. 


\section{Diskussion und Ausblick}

Die explorative Studie kann auf Basis der zirkulären und iterativen Vorgehensweise des Design-Based Research Ansatzes erste Erkenntnisse über das Gelingen von Lernprozessen von Teilnehmenden im Rahmen einer reflexionszentrierten Lernumgebung liefern. Das entwickelte, reflexionszentrierte Fortbildungskonzept ist dabei nur für diejenigen Lehrkräfte bedarfsgerecht, die bereit dazu sind, ein hohes Engagement aufzubringen, sich auf Ungewissheit einzulassen, eine forschende Haltung einzunehmen und darüber das eigene Lehrhandeln zu analysieren und weiterzuentwickeln (vgl. Ho, Watkins und Kelly 2001; Lipowsky 2009). Trifft dies zu, dann kann, wie gezeigt wurde, heterogenitätssensibles Lehrhandeln im Rahmen der Fortbildung angeregt werden. Dabei legen die Ergebnisse der Studie den Schluss nahe, dass ein Bildungs- und Transformationsprozess im Sinne Helspers angeregt werden kann, bei dem, »wenn er gelingt, man danach ein anderer geworden ist « (Helsper 2016, 104). Die Ebene des unterrichtspraktischen Handelns und die Ebene der Entwicklung von Schülerkompetenzen wurden in dieser Evaluationsstudie nicht betrachtet (vgl. Lipowsky 2009).

Um auszuschließen, dass es sich nicht doch um kurzfristige Veränderungen handelt, müssten weitere (Nach-)Erhebungen durchgeführt werden (vgl. ebd.). Dies könnte zum Beispiel in der Form einer zeitlich versetzten Interviewstudie, idealerweise verknüpft mit Unterrichtsbesuchen bei den teilnehmenden Lehrkräften, erfolgen. Die Ergebnisse geben weiter Aufschluss über verschiedene Ausprägungen heterogenitätssensiblen Lehrhandelns, die im Rahmen der Fortbildung entwickelt wurden. Für die Aus- und Fortbildung von (zukünftigen) Lehrkräften wäre es hilfreich, diese Ausprägungen theoretisch und empirisch zu fundieren, um diese (noch) gezielter fördern zu können. Dabei wäre es in weiterführenden, qualitativ-rekonstruktiven Analysen auf Fallebene vielversprechend, mögliche Verbindungen zwischen den unterschiedlichen Ausprägungen der Bewusstheit für die eigene Beobachtungs- und Handlungspraxis einerseits und der Bereitschaft der Teilnehmenden, das eigene Beobachten und Handeln weiterzuentwickeln, andererseits zu untersuchen und ggf. auch Entwicklungsstufen dabei zu rekonstruieren. Auch die Reflexionsfähigkeit der Teilnehmenden und eine mögliche Weiterentwicklung dieser im Rahmen der Fortbildung könnten analysiert und im Zusammenhang mit der Entwicklung des heterogenitätssensiblen Lehrhandelns betrachtet werden.

Die Ergebnisse dieser Design-Based Research Studie sind auf die Implementation im universitären Kontext bezogen (vgl. Raatz 2016). Dies könnte auch einen Einfluss auf die Erwartungshaltung der Teilnehmenden nach wissenszentrierten Lernumgebungen gehabt haben. Eine zentrale Rolle spielen zudem die Dozierenden mit universitärem Background: Für die Einnahme der Dozierenden-Rolle ist hier einerseits das notwendige sozialwissenschaftliche und pädagogisch-didaktische Wissen unablässig, aber andererseits auch die Fähigkeit, die Teilnehmenden bei der Einnahme der Analyseper- 
spektive anzuleiten und zu unterstützen. Hier stellt sich die Frage, inwieweit dieses Fortbildungskonzept mithilfe der entwickelten Gestaltungsprinzipien auch in andere Fortbildungskontexte wie zum Beispiel in das Fortbildungsangebot der Akademien implementiert werden könnte. Dies könnte im Rahmen einer Implementationsstudie analysiert werden. Gleichzeitig wäre es aber mit Blick auf die Professionalisierung für Inklusion ein wichtiges Anliegen, herauszufinden, in welcher Form möglichst viele Lehrkräfte bezüglich eines reflektierten Umgangs mit Heterogenität bzw. Differenz fortgebildet werden können etwa im Rahmen von Schulentwicklungsmaßnahmen.

\section{Anmerkungen}

1 Das diesem Bericht zugrunde liegende Vorhaben wurde mit Mitteln des Bundesministeriums für Bildung und Forschung unter dem Förderkennzeichen 01JA1611 gefördert. Die Verantwortung für den Inhalt dieser Veröffentlichung liegt bei den Autorinnen.

2 Gestaltungs- und anwendungsorientierte Forschungsansätze zeichnen sich durch ein exploratives Vorgehen aus, bei dem praktische und wissenschaftliche Interessen miteinander verknüpft werden sollen. Dieser Zugang ist von der empirisch-quantitativ geprägten und wirkungsorientierten Lehr-/Lernforschung abzugrenzen (vgl. Raatz 2016).

3 Für eine ausführliche Darstellung des Fortbildungskonzeptes in allen seinen Schritten und Elementen siehe Baust und Pachner (2020).

4 Die Ergebnisse der zur Abschlussevaluation genutzten Seminarmethode »Bilanzwaage« (Knoll 2007) liegen als Fotoprotokoll vor.

5 Die Fortbildung richtete sich an berufstätige Lehrkräfte der Sekundarstufe. Von den 17 angemeldeten Lehrkräften sind 14 weiblich und drei männlich. Fünf der Lehrkräfte unterrichten an Gemeinschaftsschulen, sieben an beruflichen Schulen, drei an Gymnasien und eine an einer Realschule. Ausbildung, Berufsdauer und Schulstufe der Lehrkräfte wurden nicht erhoben.

6 Von den sieben Lehrkräften, die in die Auswertung einbezogen wurden, sind drei männlich und vier weiblich. Drei der Lehrkräfte unterrichten an beruflichen Schulen, drei an Gymnasien, eine an einer Realschule.

7 Von den 17 angenommenen Lehrkräften sind zwölf weiblich und fünf männlich. Drei der Lehrkräfte unterrichten an Gemeinschaftsschulen, vier an beruflichen Schulen, sechs an Gymnasien, zwei an Realschulen und zwei an Sonderberufsschulen.

8 Von den drei Lehrkräften, die in die Auswertung einbezogen wurden, sind zwei männlich und eine weiblich. Zwei der Lehrkräfte unterrichten an Gymnasien, eine an einer Sonderberufsschule.

\section{Literatur}

Amrhein, Bettina. 2011. Inklusion in der Sekundarstufe - Eine empirische Analyse. Bad Heilbrunn: Klinkhardt.

Arnold, Rolf. 1985. Deutungsmuster und pädagogisches Handeln in der Erwachsenenbildung. Bad Heilbrunn: Klinkhardt.

Baust, Christina, und Anita Pachner. 2020. »Transformation ermöglichen. Potentiale von Reflexion und Perspektivverschränkung für die Professionalisierung von Lehrenden. Evaluationsstudie zu einem reflexionszentrierten Fortbildungsangebot der Erwachsenenbildung für Lehrkräf- 
te." In Erwachsenenbildung und Lernen in Zeiten von Globalisierung, Transformation und Entgrenzung, herausgegeben von Olaf Dörner, Carola Iller, Ingeborg Schüßler, Heide von Felden, Sebastian Lerch, 61-75. Opladen, Berlin, Toronto: Barbara Budrich.

Biederbeck, Ina, und Martin Rothland. 2017. »Professionalisierung des Umgangs mit Heterogenität.« In Umgang mit Heterogenität in Schule und Unterricht. Grundlagentheoretische Beiträge, empirische Befunde und didaktische Reflexionen, herausgegeben von Thorsten Bohl, Jürgen Budde und Markus Rieger-Ladich, 223-235. Bad Heilbrunn: Klinkhardt.

Emmerich, Marcus, und Daniel Goldmann. 2018. »Tübinger Fortbildung zu Heterogenität in Unterricht und Schule (TüFHUS).« Accessed March 4, 2019. https://uni-tuebingen.de/de/126119.

Emmerich, Marcus, und Ulrike Hormel. 2013. Heterogenität - Diversity - Intersektionalität. Zur Logik sozialer Unterscheidungen in pädagogischen Semantiken der Differenz. Wiesbaden: Springer.

Euler, Dieter. 2014b. »Design-Research - a paradigm under development." In Design-based Research. Zeitschrift für Berufs- und Wirtschaftspädagogik/Beiheft, herausgegeben von Dieter Euler und Peter F.E. Sloane, 15-41. Stuttgart: Steiner.

Flick, Uwe. 2009. „Triangulation in der qualitativen Forschung.« In Qualitative Forschung. Ein Handbuch, herausgegeben von Uwe Flick, Ernst von Kardorff und Ines Steinke, 309-318. Hamburg: Rowohlt.

Greif, Siegfried. 2008. Coaching und ergebnisorientierte Selbstreflexion. Göttingen [et al.]: Hogrefe.

Helsper, Werner. 2016. "Lehrerprofessionalität - der strukturtheoretische Ansatz." In Beruf Lehrer/Lehrerin: ein Studienbuch, herausgegeben von Martin Rothland, 103-125. Münster, New York: Waxmann.

Ho, Angela. 2000. »A conceptual change approach to staff development: a model for programme design."International Journal of Academic Development 5 (1): 30-41.

Ho, Angela, David Watkins und Mavis Kelly. 2001. »The conceptual change approach to improving teaching and learning: An evaluation of a Hong Kong staff development program."Higher Education 42: 143-169.

Hof, Christiane. 2007. „Ein empirisch fundierter Vorschlag zur Typisierung von Lernumgebungen." In Lernertypen - Lernumgebung - Lernerfolg, herausgegeben von Arnim Kaiser, Ruth Kaiser und Reinhard Hohmann, 35-59. Bielefeld: W. Bertelsmann.

Hüfner, Gerd. 2012. »Frust-Projekt Inklusion - Allein gelassen mit großen Aufgaben.« Bayerische Schule - Zeitschrift des bayerischen Lehrer- und Lehrerinnenverbands 65(5): 6-9.

Knoll, Jörg. 2007. Kurs- und Seminarmethoden - Ein Trainingsbuch zur Gestaltung von Kursen und Seminaren, Arbeits- und Gesprächskreisen. Weinheim/Basel: Beltz.

Idel, Till-Sebastian, Kerstin Rabenstein und Norbert Ricken. 2017. "Zur Heterogenität als Konstruktion. Empirische und theoretische Befunde einer ethnographischen Beobachtung von Ungleichheitsordnungen im Unterricht.«In Differenz - Ungleichheit - Erziehungswissenschaft. Verhältnisbestimmungen im Interdisziplinären, herausgegeben von Isabell Diehm, Melanie Kuhn und Claudia Machold, 139-156.Wiesbaden: Springer.

Leipziger, Eva, Tobias Tretter und Markus Gebhardt. 2012. »Inklusion an oberfränkischen Grundschulen. Inklusive Entwicklung in Bayern.« Zeitschrift für Heilpädagogik 63 (10): 433-439. Würzburg: Verband Sonderpädagogik.

Lipowsky, Frank. 2009. »Unterrichtsentwicklung durch Fort- und Weiterbildungsmassnahmen für Lehrpersonen.«In Beiträge zur Lehrerinnen- und Lehrerbildung 27 (3): 346-340.

Lipowsky, Frank. 2010. »Lernen im Beruf - Empirische Befunde zur Wirksamkeit von Lehrerfortbildung." In Lehrerinnen und Lehrer lernen. Konzepte und Befunde zur Lehrerfortbildung, herausgegeben von Florian H. Müller, 51-70. Münster: Waxman.

Mayring, Philipp A.E. 2015. Qualitative Inhaltsanalyse. Grundlagen und Techniken (12. Aufl.). Weinheim und Basel: Beltz. 
Mezirow, Jack. 1997. Transformative Erwachsenenbildung. Baltmannsweiler: Schneider Verlag Hohengehren.

Pachner, Anita. 2013. »Selbstreflexionskompetenz. Voraussetzung für Lernen und Veränderung in der Erwachsenenbildung?« Magazin erwachsenenbildung.at. Das Fachmedium für Forschung, Praxis und Diskurs, 20: 1-9. http://www.erwachsenenbildung.at/magazin/13-20/meb13-20. pdf.

Pachner, Anita. 2014. »Die Metakompetenz ıSelbstreflexion« und ihre Bedeutung für pädagogisch Tätige und deren Professionalitätsentwicklung. «Aufbruch in die Zukunft. Erfolgreiche Entwicklungen von Schlüsselkompetenzen in Schulen und Hochschulen, herausgegeben von Volker Heyse, 434-447. Münster: Waxmann.

Raatz, Saskia. 2016. Entwicklung von Einstellungen gegenüber verantwortungsvoller Führung. Eine Design-based Research Studie in der Executive Education. Wiesbaden: Springer.

Schmidt-Hertha, Bernhard, und Evamaria Werner. 2019 . „Qualität in der Lehrerfortbildung - Reflexionen anhand einer aktuellen Fortbildungsinitiative." In Qualität, Professionalisierung und Monitoring im Bildungswesen, herausgegeben von Hartmut Ditton und Rudolf Tippelt, 249-263. Münster: Waxmann.

Schön, Donald A. 1983. The reflective practitioner. How professionals think in action. New York: Basic Books.

Siebert, Horst. 2011. Selbsteinschließende Reflexion als pädagogische Kompetenz. In Veränderung durch Selbstveränderung. Impulse für das Changemanagement, herausgegeben von Rolf Arnold, 9-18. Baltmannsweiler: Schneider Verlag Hohengehren.

Siebert, Horst. 2012. Didaktisches Handeln in der Erwachsenenbildung. Didaktik aus konstruktivistischer Sicht (7. Aufl.). Augsburg: ZIEL.

Terhart, Ewald. 2015. „Umgang mit Heterogenität: Anforderungen an Professionalisierungsprozesse.« In (Keine) Angst vor Inklusion. Herausforderungen und Chancen gemeinsamen Lernens in der Schule (Münstersche Gespräche zur Pädagogik), herausgegeben von Christian Fischer, 69-85. Münster: Waxmann.

Tietgens, Hans. 1988. »Professionalität für die Erwachsenenbildung.«In Professionalität und Professionalisierung, herausgegeben von Wiltrud Gieseke, 28-75. Bad Heilbrunn: Klinkhardt.

\section{Die Autorinnen}

Christina Baust, M. A., ist wissenschaftliche Mitarbeiterin der Abteilung Erwachsenenbildung/Weiterbildung der Universität Tübingen. Ihre Arbeitsschwerpunkte sind: Professionalität und Professionalisierung von Lehrenden in Schule und Erwachsenenbildung.

Kontakt: christina.baust@uni-tuebingen.de

Anita Pachner, Prof. Dr., hat die Professur für Erziehungswissenschaft mit dem Schwerpunkt Erwachsenenbildung/Weiterbildung an der Universität Tübingen inne. Ihre Arbeitsschwerpunkte sind: Professionalität und Professionalisierung von Lehrenden in Schule und Erwachsenenbildung, (non-)formale und informelle Prozesse des Kompetenzerwerbs, Validierung von Kompetenzen, Rekonstruktion von Selbstreflexionskompetenz.

Kontakt: anita.pachner@uni-tuebingen.de 